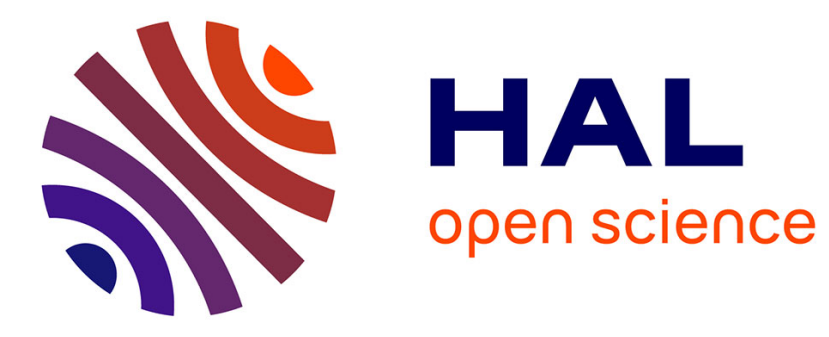

\title{
Soft Nanofluidic Transport in a Soap Film
}

Oriane Bonhomme, Olivier Liot, Anne-Laure Biance, Lyderic Bocquet

\section{To cite this version:}

Oriane Bonhomme, Olivier Liot, Anne-Laure Biance, Lyderic Bocquet. Soft Nanofluidic Transport in a Soap Film. Physical Review Letters, 2013, 110 (5), pp.054502. 10.1103/PhysRevLett.110.054502 . hal-03014155

\section{HAL Id: hal-03014155 https://hal.science/hal-03014155}

Submitted on 11 Feb 2021

HAL is a multi-disciplinary open access archive for the deposit and dissemination of scientific research documents, whether they are published or not. The documents may come from teaching and research institutions in France or abroad, or from public or private research centers.
L'archive ouverte pluridisciplinaire HAL, est destinée au dépôt et à la diffusion de documents scientifiques de niveau recherche, publiés ou non, émanant des établissements d'enseignement et de recherche français ou étrangers, des laboratoires publics ou privés. 


\title{
Soft Nanofluidic Transport in a Soap Film
}

\author{
Oriane Bonhomme, Olivier Liot, Anne-Laure Biance, ${ }^{*}$ and Lydéric Bocquet \\ Institut Lumière Matière, University Lyon 1-CNRS, UMR 5306, Université de Lyon, 69622 Villeurbanne cedex, France
}

(Received 30 October 2012; published 1 February 2013)

\begin{abstract}
We investigate experimentally the electrokinetic properties of soft nanofluidic channels that consist in soap films with nanometric thickness, covered with charged surfactants. Both the electric and fluidic responses of the system are measured under an applied voltage drop along the film. The electric field is shown to induce an electro-osmotic hydrodynamic flow in the film. However, in contrast to systems confined between solid surfaces, the soft nature of the nanochannel results furthermore in a thickening of the film. This effect accordingly increases the total electro-osmotic flow rate, which behaves nonlinearly with the applied electric field. This behavior is rationalized in terms of an analogy with a Landau-Levich film withdrawn from a reservoir, with the driving velocity identified here with the electro-osmotic one.
\end{abstract}

The development of microfluidics and nanofluidics fabrication methods has raised new challenges to drive and manipulate flows in ever more tiny channels [1]. Indeed, new behaviors arise at nanoscales where surface effects become predominant [2]. Here, we consider soft nanofluidic systems, in which the fluid is confined between soft deformable surfaces. While the production of soft matter nanoscale capsules or membranes by self-assembly of various amphiphilic species has been known for decades [3], such systems have barely been explored for nanofluidic transport characterization. In particular, little attention has been paid to electrokinetic phenomena in such controlled films. Electrokinetic properties take their origins at interfaces, where hydrodynamic flow is coupled to ion repartition. They are accordingly strongly sensitive to molecular details of the interface. In particular, hydrodynamic slip at the surface has been shown to enhance electro-osmotic (EO) flows in solid channels $[2,4,5]$. In soft fluidic channels, the deformability of the surface enters as a new parameter, which can therefore strongly modify the hydrodynamic description of the flow.

We propose to investigate these properties by characterizing electro-osmotic flow in a soap film. Soap films indeed have many advantages: They are easy to produce; their thickness is intrinsically in the nanometer range, since it results from a balance between liquid pressure and surfactant interactions [6]; and eventually, with ionic surfactants, a surface potential larger than $100 \mathrm{mV}$ may be obtained. How these specific properties affect electrokinetic transport is an open issue that we tackle here. Such effects are crucial as they are encountered in many biological systems [7] or soft membranes and emulsions.

The experimental procedure consists in confining a bubble between two horizontal electrodes made of glass slides coated with a $30 \mathrm{~nm}$ thick layer of platinum. As illustrated in Fig. 1, a cylindrical film is created that connects the electrodes via meniscii (that act as a reservoir of the foaming solution). The foaming solution consists of a tetradecyltrimethylammonium bromide, a cationic surfactant, and solution above the critical micelle concentration (Aldrich, $3 \mathrm{~g} / \mathrm{l}$ ) mixed with potassium chloride (Roth, $1 \mathrm{mM}$ ) in deionized water (Millipore). This total electrolyte concentration sets the Debye length of the system at $\lambda_{D}=5 \mathrm{~nm}$. Surface tension $\gamma$ is measured by a Whilelmyplate experiment and is equal to $36 \pm 1 \mathrm{mN} / \mathrm{m}$. The solution conductivity $K_{\text {bulk }}$ is measured with a conductimeter (Hanna instrument) and is $58 \pm 2 \mathrm{mS} / \mathrm{m}$ in the present conditions. The setup is sealed in a hermetic box with a water saturated atmosphere to avoid evaporation, and the upper electrode is slightly curved to prevent any movement of the bubble.

An electric field is generated within the soap film by applying a potential drop $\Delta V$ on the electrodes. It reads $E=-\frac{\Delta V}{h}$, where $h$ is the film height; see Fig. 1. The subsequent electrical current generated through the bubble is recorded via a homemade $I-V$ converter, controlled by Labview software National Instruments.

To characterize the EO flow in these soft channels, we investigate both the channel thickness and liquid flow rate
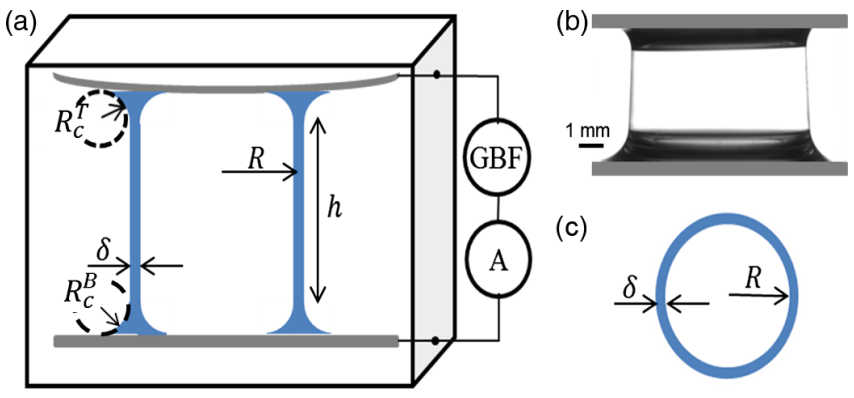

FIG. 1 (color online). (a) Scheme and notations of the experimental configuration, with the cylinder soap film (blue or light gray), the electrodes (gray or dark gray) and the contacting liquid meniscii (blue or light gray) with radii of curvature $R_{c}^{B}$ and $R_{c}^{T}$ for the bottom and top ones. (b) Picture of the soap film between the electrodes. (c) Top view with notations. 
under applied electric field in several experimental configurations. To this aim, the bubble shape is recorded (Manta Camera, Allied Vision): The film height $h$, the bubble radius $R$, and the curvature radii of the bottom and top meniscii $R_{c}^{B}$ and $R_{c}^{T}$ are measured. The film thickness $\delta$ is determined through conductivity measurement $[8,9]$. For a flat film, which is indeed in good agreement with our color observations, the soap film acts as an electrical resistance and the electrical current measured $I$ is directly linked to its thickness by the following equation:

$$
\frac{I}{\Delta V}=\frac{2 \pi R \delta}{h} K,
$$

where $K$ is the film conductivity. This conductivity has two contributions [9,10]: One, denoted as $K_{\text {bulk }}$, is due to bulk ion mobility, and the second, denoted as $K_{\text {sat }}$, originates from ionic surface transport and reads

$$
K_{\mathrm{sat}}=\mu \frac{2 \sigma}{\delta},
$$

where $\mu$ is the ionic mobility and $\sigma$ the surface charge. The surface contribution becomes dominant for channel sizes smaller than the so-called Dukhin length $l_{\mathrm{Du}}=\frac{\sigma}{e c_{0}}$ [2], where $c_{0}$ is the ion concentration. $l_{\mathrm{Du}}$ is estimated in our foaming solution as $l_{\mathrm{Du}} \simeq 120 \mathrm{~nm}$ with a surface charge of $\sigma \simeq 0.040 \mathrm{C} / \mathrm{m}^{2}$ observed for similar soap films [11]. For a precise film thickness measurement, the surface conductivity due to the surfactant layer is first determined experimentally. A $100 \mathrm{~Hz}$ ac voltage difference-in order to avoid long-time effects of electro-osmosis-is applied with a generator [low frequency generator (GBF) on Fig. 1], and the amplitude of the resulting electrical current is recorded. The continuous curve reported in Fig. 2(c) represents the time evolution of the normalized conductance $G=h I / 2 \pi R \Delta V$. Several regimes can be identified. Initially $(t<0)$, there is no film between the electrodes, and the current is thus vanishing. Once the film is created (at $t=0 \mathrm{~s}$ ), the normalized conductance first decreases quickly with time, due to thinning of the film under drainage [12]. After $100 \mathrm{~s}, G$ reaches a saturation plateau. Note that an abrupt change of this plateau value toward zero is a signature of film breakage, as is exemplified in Fig. 2. The saturation value can have different origins: Either the film thickness becomes lower than the Dukhin length and conductivity saturates at its surface value or drainage stops once the film reaches an equilibrium thickness. However, the equilibrium thickness is expected to be of order $50 \mathrm{~nm}$, as can be estimated from a balance between capillary suction by the curved contacting meniscii and the electrostatic interactions between surfactants on the film surface (using $R_{c} \approx 0.5 \mathrm{~mm}$ and the disjoining pressure isotherm found in Ref. [11]). This estimated size is at least 2 times smaller than the Dukhin length, and the plateau of the conductance is accordingly attributed to the saturating surface conductance. Moreover, the conductance obtained in such experiments is found to be independent of the
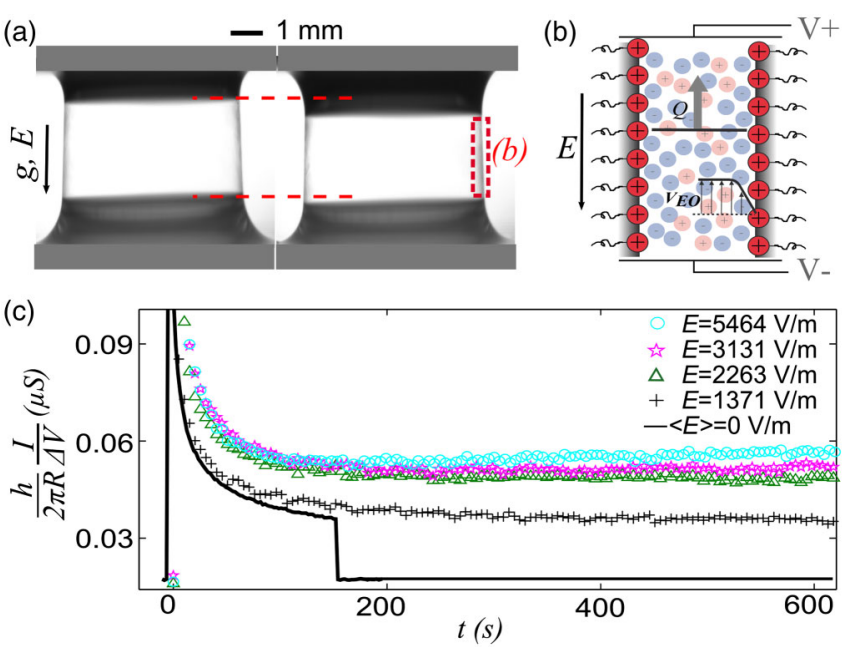

FIG. 2 (color online). (a) Pictures of a bubble under an electrical field $E=70420 \mathrm{~V} / \mathrm{m}$, separated by $200 \mathrm{~s}$. The dashed red line locates the position of the meniscus at the beginning of the experiment. (b) Zoom-in of the framed zone in (a): scheme of the soap film structure and of the electro-osmosis mechanism. (c) Geometrically normalized conductance $G$ versus time and for different applied electric fields. The continuous curve is obtained for an ac field with $\langle E\rangle=0 \mathrm{~V} / \mathrm{m}$; the symbols correspond to the dc field, as indicated in the figure.

radius of curvature of the contacting meniscus and equal to $K_{\text {surf }}=K_{\text {sat }} \delta \approx(0.77 \pm 0.04) 10^{-8} \mathrm{~S}$. This corresponds to a surface charge of $\sigma \approx 0.047 \mathrm{C} / \mathrm{m}^{2}$ [using Eq. (2) with $\mu=81 \times 10^{-9} \mathrm{~m}^{2} \mathrm{~s}^{-1} \mathrm{~V}^{-1}$ ]. This value is close to measurements on a thin film balance for tetradecyltrimethylammonium bromide in water [11]. In the following, we shall use this measured value of surface conductivity to deduce film thickness in the range of hundreds of nanometers from conductivity measurements.

In a second step, we investigate EO flow through the soap film. A dc voltage difference is applied on the electrodes to generate a constant electrical field along the film in the gravity direction. We show in Fig. 2(a) pictures of a bubble subjected to a constant electrical field for various times. We observe that a liquid flow builds up from bottom to top, which is a signature of the EO flow that occurs within the soap film. Furthermore, the top meniscus has swollen as time elapses. As surface charge is positive here, negative counterions in the Debye layer are driven by this electric field and drag the liquid within the film, as reported in Fig. 2(b).

Time evolutions of the geometrically normalized film conductance $G$ for several voltage drop amplitudes are reported in Fig. 2(c) (symbols). As in the previous situation, in the absence of a dc electric field, a first drainage regime is followed by a plateau in the conductance, whose value increases with the electric field magnitude. This shows clearly that electro-osmosis is accompanied by a film thickening. Moreover, if the electric field is suddenly modified during an experiment, a change of film color is observed as a result of thickness change. Two parameters are identified 
as affecting the film thickening: the radius of curvature of the bottom meniscus and the electric field intensity.

We perform experiments with various radii of curvature of the bottom meniscus by injecting or pumping liquid on the bottom electrode. In Fig. 3(a), we report the stationary value of the film thickness versus this radius $R_{c}^{B}$. The thickness increases linearly with $R_{c}^{B}$ and is 5-10 times larger than in the absence of an electrical field. In the following, we consider accordingly the slope $\delta / R_{c}^{B}$ as the relevant physical parameter. Film thickening is also increasing with the magnitude of the electrical field, $E$, as illustrated in Fig. 3(b). We observe that the normalized thickness varies as a sublinear power law of the electrical field, as $\delta / R_{c}^{B} \simeq(7 \pm 0.15) 10^{-7} E^{2 / 3}$.

These two dependencies can be interpreted theoretically by an analogy with the classical Landau-Levich-Derjaguin (LLD) withdrawing film problem [13]. In the original situation, a plate is withdrawn from a bath at a constant velocity $V$ and a film is deposited on the plate, whose thickness $\delta$ is fixed by a balance between viscous entrainment and capillary suction $\delta \propto R_{c}(\eta V / \gamma)^{2 / 3}$.

In our case, the film is driven by the electric forces at the surfaces, withdrawing liquid from the bottom reservoir. To describe this situation, we write the force balance within the soap film in the vertical direction, within the lubrication approximation:

$$
\eta \frac{\partial^{2} v_{z}}{\partial x^{2}}=\rho g-\frac{\gamma}{2} \frac{\partial^{3} \delta}{\partial z^{3}}+q(x, z) E,
$$

where $z(x)$ is parallel (perpendicular) to the film surfaces, $v_{z}$ is the velocity field in the $z$ direction, and $q(x, z)$ is the charge within the film, following a Poisson-Boltzmann
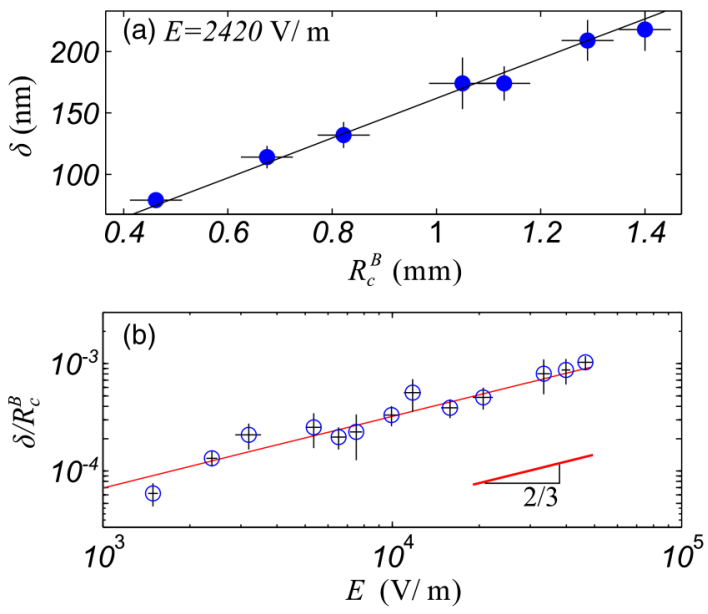

FIG. 3 (color online). (a) Film thickness $\delta$ versus the radius of curvature of the bottom meniscus $R_{c}^{B}$ for an electric field $E=$ $2420 \pm 40 \mathrm{~V} / \mathrm{m}$. The solid line is a linear fit. Each point corresponds to an average of at least five experiments in comparable conditions. (b) $\delta / R_{c}^{B}$, deduced from the previous plot, as a function of the applied electric field $E$ in a log-log plot. The solid line is a power-law fit as $\delta / R_{c}^{B} \propto E^{2 / 3}$. repartition [14]. As with the LLD problem [13], the thickness can be determined by asymptotic matching of the film with the static meniscus with a radius of curvature $R_{c}^{B}$. These equations are completed by a no-slip boundary condition at surfaces $\left(\left.v_{z}\right|_{\text {surface }}=0\right)$, assuming that the surfactants at the interface are immobile. While this hypothesis does not apply to a bare liquid gas interface, the presence of surfactants affects this boundary condition; the electric field indeed drives ions and surfactants in opposite directions, and we assume that friction between both is large enough to induce zero velocity at the border. In the thin Debye layer limit, a pluglike flow is considered for the mass contribution. We also assume a constant electric field in the vertical direction. Gravitational drainage is neglected in this calculation: Indeed, from the drainage curves with $\langle E\rangle=0 \mathrm{~V} / \mathrm{m}$, the drainage velocity is indeed estimated to a few hundred of microns per second to be compared to the electro-osmotic velocity in the millimeter per second range; see Fig. 4(b). We have solved the above equations, making use in the derivation of the analogy to the problem to LLD geometry [15]. We find accordingly that the film thickness is given by an equilibrium between the electrical forcing and capillary suction, according to

$$
\delta \simeq 2.68 R_{c}^{B}\left(\frac{\eta v_{\mathrm{EO}}}{\gamma}\right)^{2 / 3},
$$

where $v_{\mathrm{EO}}=\epsilon \zeta E / \eta$ is identified with the electro-osmotic velocity and $\zeta$ is the so-called zeta potential, commonly interpreted as the electrostatic potential at the position where the velocity vanishes [2].
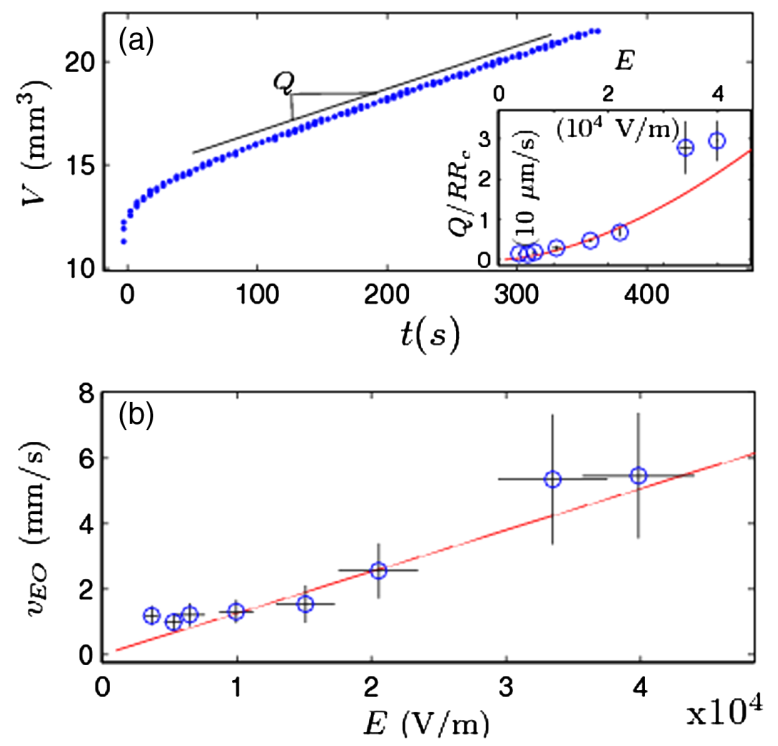

FIG. 4 (color online). (a) Volume $V$ of the upper meniscus versus time $t$. Inset: geometrically normalized flow rate (slope of the curve before) versus applied electric field E. (b) Electroosmotic velocity as a function of the applied electric field. The straight line corresponds to the electro-osmotic velocity calculated with $\zeta=160 \mathrm{mV}$. 
This law is similar to the classical Frankel law [16] where the withdrawing velocity is replaced by the electro-osmotic one. As shown in Fig. 3, this model captures the experimental dependencies of $\delta$ with both $R_{c}^{B}$ and $E^{2 / 3}$ over nearly two decades. Nevertheless, the prefactor to the experimental scaling law is overestimated by the prediction in Eq. (4) and experimentally we find $\delta=0.30 R_{c}^{B} \times \mathrm{Ca}_{\mathrm{EO}}^{2 / 3}$, with $\mathrm{Ca}_{\mathrm{EO}}=\eta v_{\mathrm{EO}} / \gamma$ and using $\zeta=130 \mathrm{mV}$, equal to the surface potential measured in such systems [11] [see also Fig. 4(b)]. However, such a discrepancy is not unexpected in view of the assumptions that were made. First, considering a constant electric field (even in the dynamic meniscus) should be examined in more detail. The stick boundary condition for the velocity at the interface should also be scrutinized and explored with other types of surfactants; indeed, the variations of the numerical prefactor in the Frankel's law versus surfactant type are still a subject of active debate [17-19]. Eventually, polarization effects near the surfaces result in deviations from continuous description, suggesting that Poisson-Boltzmann theory is questionable in these films [20]. All these issues go beyond the present work and should be investigated in the future.

Beyond film thickening, we also explore the flow efficiency of the electro-osmotic driving. To this end, the time dependent volume of the top meniscus is extracted from the variations of $R_{c}^{T}$ and monitored by image analysis (Matlab). The resulting volume variations versus time are reported in Fig. 4(a). This measurement constitutes an alternative method to extract the electro-osmotic velocity in the soap film, which complements the previous analysis of the film thickening. After a transient regime ( $\sim 20 \mathrm{~s})$, the volume varies linearly with time, confirming that the EO flow in the soap film occurs with a constant flow rate. The slopes of the curves are extracted for different applied electric fields, and the electro-osmotic flow rate is deduced, as reported in the inset of Fig. 4(a). Electro-osmotic velocity can therefore be deduced knowing the film thickness in each case. These values versus applied electric field are reported in Fig. 4(b). A linear dependency is observed experimentally and matches the Schmoluchovski prediction $v_{\mathrm{EO}}=\epsilon \zeta E / \eta$. From a linear fit, a $\zeta$ potential value of $160 \mathrm{mV}$ is measured, slightly larger than the surface potential values of $130 \mathrm{mV}$ obtained in similar systems. Furthermore, as shown in the inset of Fig. 4(a), the total flow rate $Q$ is found to scale like $Q \sim E^{5 / 3}$ in agreement with the film thickening $\delta \propto E^{2 / 3}$ established previously; see Eq. (4). This shows that, due to its deformability, the response of the soap film as a soft nanofluidic system is naturally nonlinear.

To conclude, we propose in this Letter a quantitative study of electro-osmotic flow in a soft nanochannel, a soap film. The key point of our study is that such systems are responsive and the electrodynamic response is accompanied by film thickening: Films can be up to 10 times thicker under an applied electric forcing. To our knowledge, soap film thickening by electro-osmosis has not been reported before, even if it has been observed in other situations like foam drainage [21] or foam flow [22]. We rationalize our observations with a model similar to the classical LLD pulling film theory: This model captures the dependencies of the thickening versus electric field, although overestimating it, a point that will be the object of future work. Modifying surface properties, by changing the nature of the surfactants from the mobile surfactants used here to more "rigid" ones, (for example, by addition of dodecanol to our foaming solution, according to Ref. [23]), would be particularly interesting to understand the mobility effect as well as the role of the Marangoni effect and the exchange of surfactants from bulk to surface on film thickening and flow efficiency. Furthermore, our work opens new perspectives in the context of nanofluidics, taking the benefits of the soft nature of the nanofluidic channel. Indeed, since the global flow rate is found to scale nonlinearly with electric field, $Q \propto E^{5 / 3}$, one may harvest this nonlinear response to device new fluidic functionalities, such as flow diodes. It would also be interesting to explore the regime of very thin films, where ion selectivity becomes important and nonlinear effects, such as concentration polarization and overlimiting currents, are to be expected [24].

Eventually, beyond the nanofluidic point of view, the observed effect is also very interesting in the context of liquid foam stability, for which a key mechanism at the origin of foam collapse is liquid drainage. Drainage indeed reduces the liquid fraction of the foam, which is critical for foam destabilization $[25,26]$. Reversing drainage is therefore a crucial challenge, and there are to our knowledge only few studies on this subject, all of them requiring a specific formulation of the foaming solution (addition of magnetic nanoparticles [27] or use of fatty acid tubes [28]). Our results suggest that EO flow could be used in such a structure to create a counterflow against gravity and stabilize the system, as we indeed observe at the scale of a single film. Another crucial point is that, if thickening is not accounted for, spectacular values of zeta potential would be measured, as briefly reported for differential drainage in a macroscopic foam submitted to an electric field [29]. Adapting this study to a real foam structure with films, nodes, and plateau borders [30] is a great challenge in order to use this phenomenon in real systems.

The authors acknowledge support from the ERC-AG project Micromega, Isabelle Cantat for fruitful discussions, and Jean-Michel Combes and Nicolas Terrier for technical support.

*Anne-Laure.Biance@univ-lyon1.fr

[1] T. M. Squires and S. R. Quake, Rev. Mod. Phys. 77, 977 (2005).

[2] L. Bocquet and E. Charlaix, Chem. Soc. Rev. 39, 1073 (2010). 
[3] B. Cabane and S. Henon, Liquides: Solutions, Dispersions, Émulsions, Gels (Belin, Paris, 2007).

[4] C. I. Bouzigues, P. Tabeling, and L. Bocquet, Phys. Rev. Lett. 101, 114503 (2008).

[5] M. C. Audry, A. Piednoir, P. Joseph, and E. Charlaix, Faraday Discuss. 146, 113 (2010).

[6] K. J. Mysels and M. N. Jones, Discuss. Faraday Soc. 42, 42 (1966).

[7] H. Daiguji, Chem. Soc. Rev. 39, 901 (2010).

[8] E. N. Swayne, John Newman, and C. J. Radke, J. Colloid Interface Sci. 203, 69 (1998).

[9] A. W. Reinold and A. W. Rucker, Phil. Trans. R. Soc. A 184, 505 (1893).

[10] D. Stein, M. Kruithof, and C. Dekker, Phys. Rev. Lett. 93, 035901 (2004).

[11] V. Bergeron, Langmuir 13, 3474 (1997).

[12] K. J. Mysels, K. Shinoda, and S. Frankel, Soap Films: Studies of Their Thinning and a Bibliography (Pergamon, New York, 1959).

[13] L. Landau and B. Levich, Acta Physicochim. URSS 17, 42 (1942).

[14] J. Israelachvili, Intermolecular and Surface Forces (Academic, New York, 2010), 3rd ed.

[15] See Supplemental Material at http://link.aps.org/ supplemental/10.1103/PhysRevLett.110.054502 for details on calculation.

[16] K. J. Mysels, K. Shinoda, and S. Frankel, Soap Films: Studies of Their Thinning (Pergamon, New York, 1959).

[17] E. A. Van Nierop, B. Scheid, and H. A. Stone, J. Fluid Mech. 602, 119 (2008).
[18] B. Scheid, J. Delacotte, B. Dollet, E. Rio, F. Restagno, A. A. Van Nierop, D. Langevin, and H. A. Stone, Europhys. Lett. 90, 24002 (2010).

[19] H. C. Mayer and R. Krechetnikov, Phys. Fluids 24, 052103 (2012).

[20] J. Faraudo and F. Bresme, Phys. Rev. Lett. 92, 236102 (2004).

[21] O. Pitois, C. Fritz, and M. Vignes-Adler, J. Colloid Interface Sci. 282, 458 (2005).

[22] J. Emile, E. Hardy, A. Saint-Jalmes, E. Terriac, and R. Delannay, Colloids Surf. A 304, 72 (2007).

[23] A. M. Poskanzer and F. C. Goodrich, J. Phys. Chem. 79, 2122 (1975).

[24] E. V. Dydek, B. Zaltzman, I. Rubinstein, D. S. Deng, A. Mani, and M.Z. Bazant, Phys. Rev. Lett. 107, 118301 (2011).

[25] V. Carrier and A. Colin, Langmuir 19, 4535 (2003).

[26] A-L. Biance, A. Delbos, and O. Pitois, Phys. Rev. Lett. 106, 068301 (2011).

[27] D. E. Moulton and J. A. Pelesko, Phys. Rev. E 81, 046320 (2010).

[28] A.-L. Fameau, A. Saint-Jalmes, F. Cousin, B. H. Houssou, B. Novales, L. Navailles, F. Nallet, C. Gaillard, F. Boue, and J.-P. Douliez, Angew. Chem., Int. Ed. 50, 8264 (2011).

[29] M. E. Darbello Zaniquelli and F. Galembeck, Langmuir 1, 647 (1985).

[30] D. Weaire and S. Hutzler, The Physics of Foams (Clarendon, Oxford, 1999). 